\title{
ANÁLISE DO CONSUMO MÁXIMO DE OXIGÊNIO E DA COMPOSIÇÃO CORPORAL EM ATLETAS DE FUTEBOL DA CATEGORIA SUB-20.
}

\author{
Daniel Fernando Dos Reis ${ }^{1}$, Mauro Luiz Dos Santos Junior ${ }^{1}$, Paulo Henrique Bernardino Rotta ${ }^{1}$, \\ Henrique Izaias Marcelo ${ }^{1}$, Paulo Henrique Aleixo ${ }^{1}$, Lucas Da Silva Santos ${ }^{1}$, Errisson Muniz \\ Mendes ${ }^{1}$, Marco Augusto Sada Filho ${ }^{1}$, Thiago Alves Garcia ${ }^{2}$, Guilherme Akio Tamura Ozaki ${ }^{2}$, \\ Everton Alex Carvalho Zanuto ${ }^{1}$, Robson Chacon Castoldi ${ }^{1}$, Regina Celi Trindade Camargo ${ }^{3}$, José \\ Carlos Silva Camargo Filho ${ }^{3}$.

\footnotetext{
${ }^{1}$ Universidade do Oeste Paulista - UNOESTE, Departamento de Educação Física, Presidente Prudente, SP. E-mail: fernando.daniel28@yahoo.com.br.

${ }^{2}$ Universidade Estadual de Campinas - UNICAMP. Departamento de Ortopedia e Traumatologia, Campinas, SP.

${ }^{3}$ Universidade Estadual Paulista - UNESP. Departamento de Fisioterapia, Presidente Prudente, SP.
}

\section{RESUMO}

O futebol exige grande demanda física, sendo ele fisiologicamente um esporte de grandes esforços físicos. O objetivo foi analisar a composição corporal e o VO2 máximo em um grupo de atletas de futebol da categoria sub-20 durante o Campeonato Paulista da $2^{\circ}$ divisão. A amostra foi composta por 21 atletas de faixa etária acima de 18 anos de idade, inseridos na categoria sub-20 do clube Presidente Prudente Futebol Clube. A avaliação antropométrica foi realizada por meio do método de dobras cutâneas. O VO2 Máximo foi realizado seguindo protocolo de Cooper (teste de 12 minutos). Foi verificado que os atletas percorreram distância superior a 2.800 metros e apresentaram o \%GC abaixo de $12 \%$, ainda que o \%GC não apresentou associação com o VO2 máximo $(p=0,243)$ a partir das classificações estabelecidas. Porém, apesar de mostrarem correlação inversa $(-0,170)$ para as variáveis VO2 e \%GC, não foi verificada diferença significativa entre ambas $(p=0,406)$.

Palavras-Chave: Futebol; Potência Aeróbia; VO2 máximo; Teste de Cooper; Composição Corporal.

\section{ANALYSIS OF MAXIMUM OXYGEN CONSUMPTION AND BODY COMPOSITION IN FOOTBALL ATHLETES OF THE SUB-20 CATEGORY.}

\begin{abstract}
Soccer requires a great physical demand, for being a sport that physiologically performs a big physical effort. The main of this study was to analyze the body composition and the maximum VO2 in a group of soccer athletes of the U-20 category during the Paulista Championship 2nd division. The sample consisted of 21 athletes whose ages were above 18 years and were part of the sub-20 Presidente Prudente Futebol club team. The anthropometric evaluation was performed by using the skinfold method. The maximum VO2 was performed by following the Cooper protocol (12-minute test). It was verified that the athletes crossed over 2.800 meters and presented the \%GC below $12 \%$, although the \%GC was not associated with the maximum VO2 $(p=0.243)$ from the established classifications. In addition, even though they showed an inverse correlation (-0.170) for the VO2 and \%GC variables, there was no significant difference between the two variables $(p=0.406)$.
\end{abstract}

Keywords: Soccer, Aerobic Power; VO2 maximum; Cooper test; Body composition. 


\section{INTRODUÇÃO}

O futebol é a modalidade esportiva mais popular no mundo. Nesse contexto, variáveis de treinamento como a potência aeróbia e a composição corporal, são parâmetros importantes para a realização das atividades do atleta de futebol profissional e obtenção da alta performance ${ }^{1}$.

O futebol exige uma grande demanda física, sendo ele fisiologicamente um esporte de grandes esforços físicos intermitentes e de alta intensidade ${ }^{1}$. Além disso, é um esporte de altíssima intensidade, acíclico e intermitente, no qual os jogadores percorrem cerca de $10 \mathrm{~km}$ por partida, sendo que $8 \%$ à $18 \%$ desta distância em velocidades máximas ${ }^{2}$.

O consumo máximo de oxigênio (VO2 máx), considerado como melhor indicador da capacidade de resistência cardiorrespiratória. Além disso, é um importante parâmetro preditivo de morbidades associadas, além de ser utilizado para acompanhamento e prescrição do treinamento aeróbio em atletas e indivíduos sedentários ${ }^{3}$.

O VO2 máx pode variar de $55-90 \mathrm{ml} / \mathrm{kg} / \mathrm{min}$, dependendo da modalidade desportiva ${ }^{1}$. Já as medidas antropométricas incluem avaliações de estatura, massa corporal, dobras cutâneas, diâmetros e perímetros, são essenciais para a prescrição do treinamento físico.

Para que um atleta de futebol obtenha uma melhor definição da sua aptidão física, o VO2 máx e composição corporal são dois dos principais parâmetros fisiológicos e são considerados como importantes variáveis de treinamento. O VO2 máx é uma variável que representa fisiologicamente a capacidade funcional dos sistemas respiratórios e cardiovascular, no qual é definido como a capacidade máxima do organismo captar, transportar e utilizar oxigênio ${ }^{6}$.

O desenvolvimento desta capacidade é um fator dito como determinante para melhorar a performance dos atletas em campo. Sendo assim, seu trabalho é desenvolvido de forma específica sobre o sistema aeróbio, para que haja fornecimento de energia, visando aumentar a resistência cardiovascular de cada atleta, de modo a tolerar exercícios de intensidade submáxima de longa duração.

A composição corporal é um aspecto importantíssimo para o nível de aptidão física de atletas profissionais de qualquer modalidade, visto que o excesso do tecido gorduroso age como peso indesejável em atividades como o futebol, no qual a massa corporal deve ser erguida repetidamente contra gravidade. Desse modo, pode diminuir substancialmente o desempenho ${ }^{5}$.

Para a monitoração de um período treinamento de um determinado atleta, as medidas da composição corporal é um dos métodos mais específicos e pratico para o controle de sequencias de treinamento e desenvolvimento ${ }^{6}$. As medidas da composição corporal têm sido muito importantes com frequência para treinadores e atletas, principalmente em esportes em que o nível se esforços físicos são elevados. Com as mensurações da composição corporal os atletas conseguem relacionar o comportamento do seu corpo com os programas de treinamentos elaborados pelo profissional que usa a composição corporal como ferramenta exata para a elaboração do programa de exercícios físicos ${ }^{7}$.

Sendo assim, o objetivo do presente estudo foi analisar a composição corporal e o VO2 máx em uma equipe de futebol da categoria sub-20 durante o campeonato Paulista da $2^{\circ}$ divisão. 


\section{METODOLOGIA}

\section{Abordagens de pesquisa e tipologia de pesquisa}

A abordagem de pesquisa é um estudo transversal, onde o objetivo dessa pesquisa foi obter como resultados índices numéricos para que apontam os resultados.

\section{Locais e participantes}

O teste foi realizado em 21 atletas com a faixa etária acima de 18 anos de idade, inseridos na categoria sub-20 do clube localizado na cidade de Presidente Prudente- SP.

O clube foi escolhido pelos pesquisadores por ser um time tradicional na cidade de Presidente Prudente. Os atletas foram selecionados mediante a sua inserção no clube, no qual estão participando do campeonato paulista da $2^{\circ}$ Divisão. Foram selecionados por posições, sendo elas: Goleiro (2), Zagueiro (4), Laterais (3), Meio- campo (6), Atacantes (6).

\section{Procedimentos éticos em pesquisa}

O presente estudo foi encaminhado para apreciação e parecer do comitê de ética em pesquisa em seres humanos da Universidade do Oeste Paulista - UNOESTE, e foi aprovado de acordo com CAAE 69238017.1.0000.5515. Os participantes do presente estudo receberão instrução prévia a respeito da realização das avaliações e procedimentos de execução do teste de esforço e em seguida, foram convidados a ler e assinar o Termo de Consentimento Livre e Esclarecido - TCLE.

\section{Análises antropométricas}

As medidas de dobras cutâneas foram mensuradas a partir do uso de um adipômetro da marca Cescorf com precisão de $1 \mathrm{~mm}$. Foram avaliadas as seguintes dobras: peitoral, subescapular, tricipital, abdominal, suprailíaca, coxa e panturrilha. Todas as medidas foram feitas três vezes em cada região em sistema rotacional no hemicorpo direito dos avaliados. As dobras foram mensuradas por um único avaliador experiente e o cálculo da estimativa da percentagem de gordura corporal de acordo com Jackson e Pollock ${ }^{8}$, segundo o modelo utilizado por Sangali ${ }^{9}$.

\section{Testes de VO2 máximo}

A estimativa do VO2 Máximo foi realizada seguindo o protocolo de Cooper (teste de 12 minutos). Este método de avaliação consiste em avaliar a máxima distância percorrida em 12 minutos.

Para a realização do teste, foi utilizada uma pista de 400 metros, previamente demarcada, de modo a determinar a mensuração da distância percorrida. A obtenção da variável VO2 Máximo foi determinada pela fórmula: VO2 Máximo = Distância-504 / 45.

\section{Análises estatísticas}

Após a obtenção dos valores nas avaliações, foi realizado o teste de normalidade de Shapiro-Wilk para a verificação da distribuição dos dados. Como não houve normalidade entre os dados, foi realizado a correlação de Spearman e teste do Qui-Quadrado para análise de associação entre as variáveis \%GC e o VO2 Máx. Foi admitido como diferença estatisticamente significativa valores inferiores a $5 \%(p<0,05)$. Todos os procedimentos foram realizados com $o$ Software com pacote estatístico SPSS 22.0. 


\section{RESULTADOS}

Após a análise dos dados, foi verificado que os atletas percorreram distância superior a 2.800 metros e apresentaram o \%GC abaixo de $12 \%$ (Tabela 1).

Tabela 1. Análise descritiva a partir das variáveis obtidas

Potência Aeróbia (Distância)

Média

Desvio Padrão

VO2 Máximo $\left(0^{2} / \mathrm{Kg} / \mathrm{Min}\right)$

$\begin{array}{ll}\text { Média } & 52,70 \\ \text { Desvio Padrão } & 6,35\end{array}$

Massa Corporal (Kg)

Média

71,89

Desvio Padrão

9,58

Percentual de Gordura Corporal (\%GC)

Média $\quad 11,91$

Desvio Padrão $\quad 1,52$

Foi verificado ainda que o \%GC não apresentou associação com o VO2 máximo $(p=0,243)$ a partir das classificações estabelecidas (Tabela 2).

Tabela 2. Análise de associação entre variáveis

Classificação VO2

\begin{tabular}{clllllll} 
& & Média & Superior & Excelente & Total & $X^{2}$ \\
\hline \multirow{2}{*}{ \%GC } & Média & 0 & 3 & 0 & 3 & \\
& Superior & 4 & 4 & 4 & 12 & 0,243 \\
& Excelente & 3 & 2 & 1 & 6 & \\
Total & & 7 & 9 & 5 & 21 &
\end{tabular}

Análise de associação a partir do teste do Qui-Quadrado com valor de significância de 5\% $(p<0,05)$. 
Por último, foi observado que apesar de mostrarem correlação inversa $(-0,170)$ para as variáveis VO2 e \%GC, não foi verificada diferença significativa entre ambas $(p=0,406)$.

Tabela 3. Análise de correlação entre variáveis.

\begin{tabular}{lcc}
\hline & Correlação entre variáveis \\
& $R^{2}$ & $P$ \\
\hline Classificação \%GC & 0170 & 0,406 \\
Classificação \%VO2 & & \\
\hline
\end{tabular}

Correlação de Spearman com significância de 5\% (p<0,05).

\section{DISCUSSÃO}

O futebol é uma modalidade complexa, onde a caracterização funcional de cada atleta é determinada pelo grau de intensidade e volume executado por movimentos e ações exercitas em uma partida. De modo que as respostas fisiológicas são determinadas pelo esforço de posições e funções táticas que o atleta exerce frente a um jogo ${ }^{10}$.

Diversos estudos têm se preocupado em apresentar parâmetros fisiológicos, como indicadores físicos para diferentes categorias e até mesmo por posições de cada jogador. Pinto et al 5, afirmam que as características antropométricas junto com outras ações do esporte são fatores importantes para a evolução da equipe.

Em um estudo realizado por Barbalho et al. ${ }^{11}$, ao comparar o VO2 máximo entre as posições, observou que os meio campistas obtiveram melhor resultado comparado aos de outras posições $(70,95 \mathrm{ml} / \mathrm{Kg} / \mathrm{min})$. Os laterais também obtiveram um resultado significativo em relação as outras posições $(60,46 \mathrm{ml} / \mathrm{Kg}$. $\mathrm{min})$. Destaca-se que quando comparada as outras posições entre si, goleiro $(49,46 \mathrm{ml} / \mathrm{Kg}$. $\mathrm{min})$, zagueiros $(49,39 \mathrm{ml} / \mathrm{Kg}$. min) e atacantes $(47,35$ $\mathrm{ml} / \mathrm{Kg}$. min) não houve diferença para que pudesse distingui-las.

Junior et al ${ }^{4}$., aponta que quando comparada a composição corporal e a capacidade aeróbia de diferentes posições táticas em atletas da categoria juniores, os atletas obtém características físicas e níveis de condicionamento físico distintos, decorrente das exigências metabólicas de suas funções e ações em campo.

A analise dos dados obtidos pelo grupo de jogadores apresenta uma media abaixo de $12 \%$ de percentual de gordura, sendo considerados índices de normalidade aceitáveis para futebolista desta categoria que varia de $8 \%$ á $14 \%$. O perfil encontrado entre os futebolistas deste estudo pode ser observado em outros estudos que apresentam médias da idade, peso corporal, percentual de gordura, o que permite observar um parâmetro das variáveis em futebolistas da categoria.

Relacionando com o estudo realizado por Schulz ${ }^{1}$, que apresenta uma media geral de $9,9 \% \pm 1,07$ de percentual de gordura em jogadores de futebol da categoria sub-18, mas que é vista uma variável quando comparada por posições, observamos que encontram-se dentro das características antropométricas consideráveis bom para o esporte especifico. Garret e colaboradores $^{12}$ afirmam que para o sucesso no futebol, valores de 8 a $12 \%$ são considerados para um bom índice no desporto especifico.

Podemos ainda observar algumas limitações neste estudo, por causa de poucas referencias encontradas em relação a essa categoria especifica dentro do esporte no Brasil. Porém o presente estudo sobre o VO2 máx e a composição corporal atribui gradativamente para os atletas futebolistas no desenvolvimento das capacidades envolvidas. 


\section{CONCLUSÃO}

Conclui-se que os atletas apresentam baixo percentual de gordura. Além disso, nos valores achados do VO2 máximo, o nível de aptidão física dos atletas se encontra com os valores acima da média da geral da população. 


\section{REFERÊNCIAS}

1. SCHULZ ST, SILVA JVP, NUÑEZ PRM. Análise da composição corporal e VO2 máx. em jogadores de futebol da categoria sub-18. CPEF 2009, 8(5): 127-132.

2. SHULTZE IS, LIBERALI R. Caracterização do futebol: distância percorrida, VO2máx e percentual de gordura do futebolista: revisão sistemática. RBNE 2011, 5 (29): 442-454.

3. JÚNIOR C, CESAR P, SOUZA B, RIBEIRO F, REGINA S. Análise e correlação entre o VO2 máx e a frequência de atividade física em adolescentes. ConScientiae Saúde 2012, 11 (1): 2428.

4. JUNIOR FÂP, CRESCENTE LAB, CARDOSO M, SIQUEIRA OD. VO2 máximo e composição corporal em atletas de futebol da categoria juniors. EFD 2010, (151): 1-7. http://www.efdeportes.com/efd151/VO2-maximo-em-atletas-de-futebol.htm.

5. PINTO MR, AZEVEDO VB, NAVARRO F. Alterações da composição corporal de jogadores profissionais de futebol do rio preto esporte clube. RBNE 2007, 1 (4): 17-24.

6. MAUGHAN R, BURKE L. NE. São Paulo: Artmed, 2007.

7. CAMPBELL B, SPANO M. Guia da NSCA para nutrição no exercício e no esporte. 1.ed. São Paulo: Phorte, 2015.

8. JACKSON A, POLLOCK ML. Generalized equations for predicting body density of men. Journal of Nutrition 1978, 40 (3): 497-504, https://doi.org/10.1079/BJN19780152.

9. SANGALI, Eduardo Bernardo et al. Comparison among different methods for the estimation of body fatness in brazilian elite cyclists. REF/UEM 2012, 23 (3): 355-360.

10. BALIKIAN $P$, et al. Consumo máximo de oxigênio e limiar anaeróbio de jogadores de futebol: comparação entre as diferentes posições. RBME 2002, 8 (2): 32-36.

11. BARBALHO MSM, NOVOA HJ, AMARAL JC. Consumo máximo de oxigênio (vo2) em atletas de futebol profissional de diferentes posições de jogo. RBFF 2017, 9 (32): 37-41. Jan/Abril. 2017. Disponível em: <www.rbff.com.br>

12. Garret JR, William E, Kirkendall DT. A ciência do exercício e dos esportes. Porto Alegre: Artmed, 2003. 